\title{
IMITATION AND SOCIAL LEARNING
}

\author{
SUREN BASOV ${ }^{1}$
}

1The University of Melbourne, Victoria 3010, Australia.
s.basov@unimelb.edu.au 


\section{NOTATIONS}

For any twice continuously differentiable $h: R^{n} \rightarrow R$ denote:

$$
\begin{gathered}
\nabla h=\left(\frac{\partial h}{\partial x_{1}}, \ldots, \frac{\partial h}{\partial x_{n}}\right) \\
\operatorname{divh}=\sum_{i=1}^{n} \frac{\partial h}{\partial x_{i}} \\
\Delta h=\sum_{i=1}^{n} \frac{\partial^{2} h}{\partial x_{i}^{2}} .
\end{gathered}
$$




\section{INTRODUCTION}

Interactions-based models are becoming increasingly popular in economics. A pioneering paper in this area is Föllmer (1974). For a review of recent theoretical developments and applications, see Blume and Durlauf (2000). The basic departure of this approach from the conventional one is its focus on direct rather than on market mediated interdependences between economic actors. In an interaction-based approach it is achieved by introducing social preferences which allows the construction of a precise mathematical mapping from interaction structures to associated population-wide distributions.

Though the interactions-based approach departs from conventional economic modelling in the way it models preferences, it retains the assumption of full rationality. Another way to model direct interdependence of choices is to assume individualistic preferences but relax the rationality assumption. In this case the interdependence will be produced by a social learning rule.

One can imagine a wide variety of social learning rules. Experimental studies on the acquisition of skills by humans and chimpanzees indicate a complex mix of imitation, other forms of social learning, and individual learning (Whiten et. al., 1996). Social learning is often held responsible for

cultural variations among different groups of humans. However, little has 
been done in the direction of formal modelling of social learning, especially in the economically interesting environments. A notable exception is Gale and Rosenthal (1999).

In this paper I consider a population of individuals facing a problem of choosing an alternative from a given set repeatedly over time. I analyze a particular form of learning which combines individualistic adjustment with imitation. I derive a master equation describing the evolution of the density of choices in the population and study its properties.

I address the question of costs and benefits that arise from the ability to imitate. Imitation facilitates convergence to a global optimum if it is unique, but it can prevent convergence to the optimum in more complex environments.

Another important learning mechanism, apart from the individualistic adjustment and imitation, is experimentation. Basov (2001) argues that any learning rule can be reduced to a combination of these three under some regularity conditions. Experimentation plays no role in this paper, except for as a steady state selection device.

The paper is organized in the following way. Section 2 introduces the behavioral rule and derives the master equation. Section 3 studies some prop- 
erties of the master equation and compares them with the results that would be obtained under individualistic adjustment only. Section 4 concludes.

\section{THE EVOLUTION OF THE DENSITY OF CHOICES IN A POPULATION OF IMI- TATING INDIVIDUALS}

Assume that an individual is faced with a repeated choice over time from the set of alternatives $\Omega$, which is assumed to be a compact, convex subset of $R^{L}$. The utility an individual obtains from choosing $x \in \Omega$ is given by a twice continuously differentiable function $U(x)$. Assume that all critical points of $U(\cdot)$ are interior points of $\Omega$. The individual starts by choosing some point $x_{0} \in \Omega$ and adjusts it at times $k \Delta t$, where $k$ is a natural number and $\Delta t$ is some fixed time interval. Other individuals are making similar decisions simultaneously, and, although their decisions do not affect each other directly, they are assumed to carry relevant information. To describe the adjustment rule of an individual, it is necessary to specify both the information available to the individual at the moment of adjustment and the rules of information 
processing.

I will assume that the individual knows the gradient $\nabla U\left(x_{t}\right)$ of the payoff function at the point of her current choice and observes a choice of a randomly chosen member of the population, $y_{t}$. Then she adjusts her choice according to:

$$
x_{t+\Delta t}-x_{t}=\left(\nabla U\left(x_{t}\right)+\nu\left(y_{t}-x_{t}\right)\right) \Delta t,
$$

where $\nu \geq 0$. In (1) the first term on the right hand side represents the gradient dynamics, while the second captures the idea of imitation. Gradient dynamics says the individuals adjust their choices in the direction of the steepest increase of the payoffs. Imitation means that the agent moves in the direction of the observed choice.

I am going to study the continuous time limit of the dynamics generated by (1). To pass to this limit, I have to introduce a result from the theory of stochastic processes.

Let $\xi(\cdot)$ be a stochastic process and $g(\xi, t)$ is the probability density function of $\xi$ at time $t$. If $K \subset \Omega$ is a Borel set, denote by $G(\Delta t, \xi, K)$ the 
probability of getting to the set $K$ from point $\xi$ during the time interval $\Delta t$ under the dynamics (1). Let $\langle a, b\rangle$ denote the inner product of the vectors $a$ and $b$, and let $n(\xi)$ be the unit vector orthogonal to the boundary $\partial \Omega$ of the set $\Omega$ at point $\xi$. For any $\eta>0$ let $V_{\eta}(\xi)$ denote an $\eta$-neighborhood of the point $\xi$ and $V_{\eta}^{c}$ is its complement. The following result is well known in the theory of stochastic processes:

Theorem 1 Suppose there are functions $\zeta(\xi)$ and $\Xi(\xi, t)$ twice continuously differentiable on the interior of $\Omega$ and continuously differentiable on $\Omega$ such that for any $\eta>0$ :

1. $G\left(\Delta t, \xi_{t}, U_{\eta}^{c}\right)=o(\Delta t)$

2. $\int_{U_{\eta}}\left(w_{t}-\xi_{t}\right) G\left(\Delta t, \xi_{t}, d w_{t}\right)=\zeta(\xi) \Delta t+o(\Delta t)$

3. $\int_{U_{\eta}}\left(w_{t}-\xi_{t}\right)\left(y_{t}-\xi_{t}\right)^{T} G\left(\Delta t, \xi_{t}, d w_{t}\right)=\Xi(\xi, t) \Delta t+o(\Delta t)$.

Then the function $f(\xi, t)$ is governed by the following partial differential equation:

$$
\frac{\partial f}{\partial t}+\operatorname{div}(\zeta(\xi) g)=\frac{1}{2} \operatorname{Tr}\left(D^{2}(\Xi(\xi, t) g)\right)
$$

and a boundary condition 


$$
\left\langle\zeta(\xi) g-\frac{1}{2} \nabla(\Xi(\xi, t) g, n(\xi))\right\rangle=0
$$

For a proof see Kanan (1979). Applying Theorem 1 to behavioral rule (1) and denoting by $z$ the current mean of the population choices one arrives at the following result:

Theorem 2 When the individual's adjustment rule is given by (1) and each individual is adjusting her choice independently, ${ }^{2}$ then the population density of choices $f(x, t)$ satisfies:

$$
\frac{\partial f}{\partial t}+\operatorname{div}((\nabla U(x)+\nu(z-x)) f)=0
$$

and a boundary condition:

$$
f(x, t)\langle\nabla U(x)+\nu(z-x), n(x)\rangle=0 \text { on } \partial \Omega .
$$

\footnotetext{
${ }^{2}$ For a discussion of the notion of independence of continuum of random variables, see Judd (1985).
} 
Proof. To prove the theorem one has to check conditions of Theorem 1. First, note that since $\Omega$ is compact and $\nabla U(x)$ is continuous, $\nabla U(x)+\nu(y-x)$ is bounded, that is there exists $M>0$ such that $\|\nabla U(x)+\nu(y-x)\| \leq M$. Then for any $\Delta t<\eta / M$ the probability to get out of an $\eta$-neighborhood of $x$ is

$$
G\left(\Delta t, x_{t}, V_{\eta}^{c}\right)=0=o(\Delta t)
$$

A straighforward computation shows that:

$$
\begin{gathered}
\int_{V_{\eta}}\left(w_{t}-x_{t}\right) G\left(\Delta t, x_{t}, d w_{t}\right)=(\nabla U(x)+\nu(z-x)) \Delta t . \\
\int_{V_{\eta}}\left(w_{t}-x_{t}\right)\left(w_{t}-x_{t}\right)^{T} G\left(\Delta t, x_{t}, d w_{t}\right)=o(\Delta t) .
\end{gathered}
$$

Comparing (6)-(8) with the conditions of Theorem 1 proves Theorem 2. 


\section{THE LONG-RUN BEHAVIOR OF THE POPULATION DENSITY OF CHOICES}

In this Section I study the long-run behavior of the density of choices when its time evolution is governed by (4)-(5). For this purpose one has to characterize the steady states of system (4)-(5). An important distinction is between steady states with a singleton support which is a local extremum of the utility function, and other steady states with a non-singleton support. The steady states of the first type are common for the pure gradient dynamics and the gradient dynamics with imitation. Non-singleton steady states, however, differ considerably in the cases of the gradient dynamics with and without imitation. Under pure gradient dynamics, non-singleton steady states have a support which is a subset of the set of critical points of the utility, while when imitation is introduced at least some points in the support fail to be critical points of the utility function. The above discussion can be summarized in a theorem:

Theorem 3 Let $F(x)$ be a steady state c. d. f. of stochastic process (1) and let $\nu>0$ and it is not an eigenvalue of the Hessian of $U$ for any $x \in \Omega$

- Then $F(\cdot)$ has a discrete support. If the support is a signleton, then it is 
a critical point of the utility function and vise versa any distribution whose support is a singleton which is a critical point of the utility is a steady state. If the support is not a singleton then it always contains points which are not local extrema of the payoff function.

Proof. Note that if $F(x)$ is a steady state c.d.f. with a density $f(x)^{3}$ then

$$
(\nabla U(x)+\nu(z-x)) f(x)=0 .
$$

First, note that since $\nu$ is not an eigenvalue of the Hessian of $U$ for any $x \in \Omega$ all roots of the equation

$$
\nabla U(x)+\nu(z-x)=0
$$

are isolated. But then there are only finitely many solutions to system (10). Indeed, assume to the contrary, that the set of solutions to system (10) is infinite. Denote this set by $X$. Since $X \subset \Omega$ and $\Omega$ is compact, the set $X$

\footnotetext{
${ }^{3}$ If $F(x)$ is not differentiable than $f(x)$ is distribution rather than function. In this case it should be understood as a generalized solution of (4)-(5).
} 
has a limit point in $\Omega$, denote it $x^{*}$. Let $\left\{x_{n}\right\}_{n=1}^{\infty}$ be a sequence, such that $x_{n} \in X$ for $\forall n \in N$ and $x_{n} \rightarrow x^{*}$. Then:

a. $\nabla U\left(x^{*}\right)+\nu\left(z-x^{*}\right)=0$ (by continuity)

b. $\forall \varepsilon>0 \exists n_{0} \in N$ such that $\forall n>n_{0}\left\|x_{n}-x^{*}\right\|<\varepsilon$

c. $\nabla U\left(x_{n}\right)+\nu\left(z-x_{n}\right)=0$.

But (a)-(c) imply that $x^{*}$ is a solution to (10) which is not isolated. This proves that set $X$ is finite. Let $X=\left\{x_{1}, \ldots, x_{k}\right\}$. Then the general solution to $(9)$ is

$$
f(x)=\sum_{j=1}^{k} \alpha_{j} \delta\left(x-x_{j}\right)
$$

where $\delta(\cdot)$ is Dirac's delta-function and $\alpha_{j}$ are arbitrary constants. Since $f(x)$ is a p.d.f., $\alpha_{j}$ is the probability that $x$ takes the value $x_{j}$, hence

$$
\alpha_{j} \geq 0, \quad \sum_{j=1}^{k} \alpha_{j}=1 .
$$

System (4)-(5) implies that the mean choice $z$ and the covariance matrix of choices $v$ evolve according to 


$$
\begin{aligned}
& \frac{d z}{d t}=E(\nabla U(x)) \\
& \frac{d v}{d t}=2\left(-\nu v+E\left((x-z)^{T} \nabla U(x)\right) .\right.
\end{aligned}
$$

The expectation is taken with respect to the current density. First, assume that the steady state support is a singleton. Then equation (13) implies that $\nabla U(x)=0$, hence, it is a critical point of the utility function. The proof of the inverse statement is straightforward. Now, assume that all points in the support of the steady state are local extrema of the utility function. Then equation (14) implies that $v=0$ and, hence, the support is a singleton.

Imitation is capable of producing conformist like behavior, even though there is no social pressure to confirm.

Example 1 Consider a situation where individuals have no explicit preferences for different outcomes, that is $U(x)=$ constant. Under pure gradient dynamics any distribution is a steady state, hence, the initial distribution of choices will be preserved. In the presence of imitation, however, any initial distribution will converge to a degenerate one, concentrated at the population mean. 
In this example the long-run outcomes under gradient dynamics with and without imitation were different but the difference was innocuous, since the utility was constant. The next two examples are to illustrate some potentially harmful effects of imitation. Theorem 3 guarantees that if non-singleton steady states exist they necessarily include suboptimal choices on the part of the population. However, the question of existence of such steady states remains open. To demonstrate their possibility consider the following example.

Example 2 Let $\Omega=[0,4 / 3], U(x)=x^{3}-2 x^{2}+x, \nu=7 / 5$. Then it is straightforward to check that

$$
f(x)=\frac{13}{21} \delta\left(x-\frac{3}{5}\right)+\frac{8}{21} \delta\left(x-\frac{6}{5}\right),
$$

is a steady state density function.

In the previous example $x$ can be interpreted as an effort, $x^{3}+x$ as the output and $2 x^{2}$ as the cost of effort. The steady state can be interpreted as a segregation of the population into two groups: "hardworking" type choosing effort $6 / 5$ and "relaxed" type choosing effort level $3 / 5$. Observe that fully rational individuals would have chosen either effort level $1 / 3$ or 
the effort level $4 / 3$. The same result would have been achieved under pure gradient dynamics. It means that segregation is less drastic under imitation. Similar results can be achieved if one assumes the existence of the pressure to conform, that is postulates that the individual's utility has a form

$$
U(x)=u(x)-\frac{\nu}{2}(x-z)^{2} .
$$

The first term on the right hand side expresses individualistic preferences, while the second is the disutility from deviation of the population mean. The rationale provided by this model, however, is quite different. It is also worth noting that relaxed type would be better off is they decrease the effort while hardworking type will be better off from increasing the effort. Relaxed types get higher utility than hardworking ones in this steady state.

The next example shows that a local minimum can be a Lyapunov stable steady state of the population dynamics if the imitative component is strong enough.

Example 3 Let $\Omega=[0,4]$, the utility function be

$$
U(x)=-4 x+x^{2}
$$


and $z(0)=2$. Then equation (13) implies that $z(t)=2$ at any $t \geq 0$. Suppose $\nu>2$, then the steady state distribution which gives probability one to the outcome $x=2$ is locally asymptotically stable. First, note that since $x=2$ is a local minimum of $U(x)$, by Theorem 3 it is a steady state. To prove stability consider a function

$$
L(t)=\int_{\Omega}\left(U(x)-\frac{\nu}{2}(x-2)^{2}\right) f(x, t) d x .
$$

Since it is maximized at $x(t)=2$ and its derivative evaluated along the solutions of the master equation is

$$
L^{\prime}(t)=\int_{\Omega}\left(U^{\prime}(x)-\nu(x-2)\right)^{2} f(x, t) d x>0,
$$

it is a Lyaponov function and stability is proven.

As demonstrated by these examples, introducing imitation on the top of gradient dynamics changes long-run behavior in a non-trivial way. Under pure gradient dynamics the support of any stationary distribution is the subset of the set of critical points of the utility and a stationary distribution is 
Lyapunov stable if and only if only local maxima get positive probabilities. ${ }^{4}$ Both of these results may fail if imitation is present. This implies that imitation can have rather undesirable consequences. The legitimate question is what is imitation good for. To answer this question consider the rate of change of expected utility in a population. Define

$$
V(t)=\int_{\Omega} U(x) f(x, t) d x
$$

Then, using (4)-(5), it is straightforward to show that

$$
\frac{d V}{d t}=E_{t}\|\nabla U(x)\|^{2}+\operatorname{cov}_{t}(x-z, \nabla U(x))
$$

where the expectation and the covariance are taken with respect to the current distribution of choices. Assume that $U(x)$ is strictly concave and $x^{*}$ is its unique global maximizer and let $z(0)=x^{*}$. Then (13) implies that $z(t)=x^{*}$ at any $t \geq 0$. Since under this assumptions $\operatorname{cov}_{t}(x-z, \nabla U(x))>0$, equation (20) implies that the rate of increase of expected utility is increased by imitation.

\footnotetext{
${ }^{4}$ These steady states are also Lyapunov stable in the presence of imitation. For a proof see Basov (2001).
} 
As we have seen, multiple steady states are possible and the concept of Lyapunov stability does not allow for a unique selection. To solve the selection problem one has to use the concept of stochastic stability (Freidlin and Wentzel, 1984). For this purpose replace equation (1) with

$$
x_{t+\Delta t}-x_{t}=\left(\nabla U\left(x_{t}\right)+\nu\left(y_{t}-x_{t}\right)\right) \Delta t+\varepsilon_{t}, \varepsilon_{t} \sim N\left(0, \sigma^{2} \Delta t\right) .
$$

A steady state is stochastically stable the fraction of time it spends in its arbitrary small neighborhood converges to one as $\sigma^{2}$ goes to zero. To analyze stochastically stable steady states of the process (1) one has first to establish the population dynamics for the process (21). It is given by the following theorem:

Theorem 4 Let individual adjustment rule is given by (21) and each individual adjusts her choice independently. Then the population density of choices satisfies:

$$
\frac{\partial f}{\partial t}+\operatorname{div}((\nabla U(x)+\nu(z-x)) f)=\frac{\sigma^{2}}{2} \Delta f
$$


and a boundary condition

$$
\left\langle(\nabla U(x)+\nu(z-x)) f-\frac{\sigma^{2}}{2} \nabla f, n(x)\right\rangle=0 .
$$

The proof of this theorem is similar to the proof of Theorem 2 and is omitted. The next theorem characterizes steady states of the process (21) for positive values of $\sigma^{2}$.

\section{Theorem 5 Let}

$$
f(x)=\frac{\exp \left(\frac{2}{\sigma^{2}}\left(U(x)-\frac{\nu}{2}(x-z)^{2}\right)\right.}{\int_{\Omega} \exp \left(\frac{2}{\sigma^{2}}\left(U(w)-\frac{\nu}{2}(w-z)^{2}\right) d w\right.},
$$

where $z$ solves

$$
z=\frac{\int_{\Omega} x \exp \left(\frac{2}{\sigma^{2}}\left(U(x)-\frac{\nu}{2}(x-z)^{2}\right) d x\right.}{\int_{\Omega} \exp \left(\frac{2}{\sigma^{2}}\left(U(x)-\frac{\nu}{2}(x-z)^{2}\right) d x\right.} .
$$

Then $f(x)$ is a Lyapunov stable stationary solution of (22)-(23). Moreover, all solutions to (22)-(23) have the form (24)-(25).

Proof. Direct substitution of (24) into (22)-(23) proves that it is a solution for any constant $z$. If $z$ satisfies equation $(25)$ then it is the mean of 
the distribution with density (24) and, hence, $f$ solves (22)-(23). To prove stability, consider

$$
L(t)=\int_{\Omega}\left(U(x)-\frac{\nu}{2}(x-z)^{2}-\frac{\sigma^{2}}{2} \log f\right) f d x .
$$

Evaluating $L^{\prime}(t)$ along solutions to (22)-(23) one finds

$$
L^{\prime}(t)=\int_{\Omega}\left(\nabla U(x)+\nu(z-x)-\frac{\sigma^{2}}{2} \nabla \ln f\right)^{2} f d x \geq 0
$$

It is straightforward to observe, that $L^{\prime}(t)=0$ if $f(x)$ is given by (22)-(23). Hence, $L(t)$ is Lyapunov function and stability is proven.

Let $f(x)$ be a stationary solution to (22)-(23), then

$$
z=\int_{\Omega} x f(x) d x
$$

But for a given $z$ the unique stationary solution to (22)-(23) is given by (24) (Freidlin, M., and Wentzel, 1984).

Corollary 6 There exists a stationary Lyapunov stable solution to (22)(23). 
Proof. It is sufficient to prove that (25) has a solution. Then, according to Theorem 5 , the stationary solution to (22)-(23) is given by (24).

Write (25) in a form:

$$
z=G(z)
$$

Function $G(\cdot)$ is continuous and for any $z \in \Omega(25)$ defines $G(z)$ as the expectation of a random variable with support $\Omega$. Since $\Omega$ is convex, compact subset of $R^{n}$ this implies that $G(z) \in \Omega$. Hence, by Brower fixed point theorem, $G(z)$ has a fixed point.

Let $z$ be given by (25) and define

$$
M(z)=\left\{x^{*}: x^{*} \in \arg \max _{x \in \Omega, z=G(z)}\left(U(x)-\frac{\nu}{2}(x-z)^{2}\right)\right\} .
$$

Note that if the utility function is continuous and strictly concave and $\Omega$ is compact, $M(z)$ is a singleton set. Its only element is the global maximizer of function $U(x)$. Then as $\sigma^{2} \rightarrow 0$ the probability the stationary distribution puts on arbitrary small neighborhood of one of the sets $M(z)$ converges to 
one. The stochastically stable set is given by

$$
M=\cup_{z \in \arg \max U(x)} M(z) .
$$

For a proof, see Freidlin and Wentzel (1984).

\section{DISCUSSION AND CONCLUSIONS}

In this paper I developed a formal model of boundedly rational imitation. It was shown that imitation is capable of producing social norms and decreasing variance in choices between different groups in society. These effects are similar to ones that would have been obtained under some forms of social preferences. This raises an issue about the possibility about distinguishing these modes of behavior empirically. The ability to distinguish them can have important policy implications. For example, if people imitate their smoking peers because they are boundedly rational then an education campaign explaining the smoking hazards can reduce the amount of cigarettes people smoke. However, if the reason is some form of social preferences, then the campaign is doomed to be futile.

Another important question is what imitation is good for, in other words, 
why did it evolve? On the positive side is the fact that under some circumstances imitation facilitates convergence to the optimum, on the negative that it is capable of producing non-desirable outcomes. The last result suggest that the drawbacks of imitation can be cured if it is blended with some degree of experimentation. However, it can be shown that the relaxation time of the initial distribution to the steady state is of the order of $1 / \sigma^{2}$, hence, there is a trade-off between the level of the expected utility in the steady state and the time of convergence. As long as $\sigma$ is sufficiently small, all negative effects of imitation remain at least in the short-run.

One way to try to overcome these difficulties is to tie the rate of experimentation to the characteristics of the current distribution of choices. This can be achieved, for example, by imitation of scope. It makes the experimentation rate an increasing function of the population variance, and, hence, reflects to a certain degree the trade-off between fast convergence and long-run payoffs. However, it turns out that there does not exist a continuous stochastic adjustment rule uniformly optimal across different environments. For a more detailed discussion of these questions see Basov (2001). 


\section{REFERENCES}

Basov, S. Bounded Rationality, Reciprocity, and Their Economic Consequences, Ph.D. Thesis, The Graduate School of Arts and Sciences, Boston University, 2001.

Blume, L. E., and S. N. Durlauf The Interactions-Based Approach to Socioeconomic Behavior, unpublished draft, 2000.

Föllmer, H. "Random Economies with Many Interacting Agents," Journal of Mathematical Economics, 1, pp.51-62, 1974.

Freidlin, M., and Wentzel. Random Perturbations of Dynamical Systems. New York: Springer, 1984.

Gale, D., and R. W. Rosenthal. "Experimentation, Imitation, and Stochastic Stability," Journal of Economic Theory, 1999, 84, pp.1-40.

Judd, K. L. "Law of Large Numbers," Journal of Economic Theory, 1985, 35, pp. 19-25.

Kanan, D. An Introduction to Stochastic Processes, Elsevier North Holland, Inc., 1979.

Whiten, A., Goodball, J., McGrew, W. C., Nishida, T., Reynolds, V., Sugiyama, Y., Tutin, C. E. G., Wrangham, R. W., and C. Boesch, C. "Cultures in Chimpanzees," Nature, 399, pp. 682-85, 1999. 


\section{University Library}

\section{- M M N E R VA A gateway to Melbourne's research publications}

Minerva Access is the Institutional Repository of The University of Melbourne

Author/s:

BASOV, S

Title:

Imitation and social learning

Date:

2002

Citation:

BASOV, S. (2002). Imitation and social learning. Department of Economics, The University of Melbourne.

Persistent Link:

http://hdl.handle.net/11343/33613 